\title{
STARDUSTS IN SUPERNOVA REMNANTS SEEN BY AKARI
}

\author{
Bon-Chul KoO \\ Department of Physics and Astronomy, Seoul National University, Seoul 151-747, Korea \\ E-mail:koo@astro.snu.ac.kr
}

(Received July 15, 2012; Accepted August 15, 2012)

\begin{abstract}
Supernovae (SN) and supernova remnants (SNRs) play a major role in the life-cycle of interstellar dusts. Fast shock waves generated by SN explosions sweep out the interstellar space destroying dust grains and modifying their physical and chemical properties. The dense, cooling SN ejecta, on the other hand, provide an environment for dusts to condense. Recent space-infrared telescopes have revealed the hidden universe related to these fascinating microscopic processes. In this paper, I introduce the results on stardusts in young core-collapse supernova remnants obtained by AKARI. The AKARI results show diverse infrared characteristics of stardusts associated with SNRs, implying diverse physical/chemical stellar structures and circumstellar environments at the time of explosion.
\end{abstract}

Key words: circumstellar matter; dust; infrared: stars; ISM: supernova remnants

\section{INTRODUCTION}

The interstellar dust that we see today is the result of long-term evolution, after many cycles of stardust injection into interstellar space followed by destruction, erosion, growth, and coagulation. SN explosions play a major role in this dust evolution or dust life cycle. First of all, the strong shocks that these explosions generate sweep out the interstellar space and destroy dust grains at a rate of $\sim 0.1 M_{\odot} \mathrm{yr}^{-1}$ (Draine, 2009), which is the main process for dust destruction. But, at the same time, dusts can condense in cooling, metal-rich SN ejecta and injected in the interstellar space. Which dust species and how much dusts form, however, are rather controversial. Theoretically, as much as $1 M_{\odot}$ of dusts can form, and in 1987A and Cas A, a comparable amount has been detected (Nozawa, 2010; Matsuura, 2011; Sibthorpe, 2011). But in other SNe/SNRs, the reported amount of SN dusts is very small. Depending on the dust injection rate, SN may or may not be the major source in the dust inventory of galaxies (cf. Draine, 2009).

The SNRs in the Galaxy and nearby galaxies, because of their proximity, provide an invaluable op- portunity to study both the microscopic processes in dust/shock physics and also the role of SN explosions in the dust life cycle. We have been doing infrared studies of SNRs using the AKARI infrared space telescope. Infrared observations are essential for studying the dust-related phenomena in SNRs because both newlyformed and blast-wave-swept-up dust grains emit essentially all of their energy in the infrared. We did pointed observations of 17 Galactic SNRs as part of ISMGN (PI. Kaneda). Most observations were IRC imaging observations to benefit from the unique S11/L15 bands and the large $\left(10^{\prime} \times 10^{\prime}\right)$ field-of-view of AKARI, but some IRC spectroscopic observations were also performed (Shinn et al., 2011 and references therein). We have also studied SNRs in the Large Magellanic Cloud (LMC) using the AKARI LMC survey data, and we are now exploring the far-infrared (FIR) properties of the Galactic SNRs using the FIS all-sky survey data. Some of the results are presented in this workshop. For the general content of the AKARI SNR project, the readers may refer to the paper by Koo (2009).

In this paper, I introduce the results of the AKARI observations of dust emission in Galactic 
young core-collapse (CC) SNRs; Cassiopeia A (Cas A), G292.0+1.8, G54.1+0.3, and MSH 15-52. In young CC SNRs, we can observe not only newly-formed dusts in SN ejecta but also circumstellar (CS) dusts that the massive progenitors injected during their final stages of evolution. By observing these stardusts, we can learn the final evolution of massive stars as well as the formation and destruction of dust grains in SNRs. I also introduce a recent AKARI result on very young, extragalactic SNRs, which showed the potential of future infrared instruments.

\section{STARDUSTS IN SUPERNOVA REMNANTS SEEN BY AKARI}

2.1. Cas A: SNR with a Large Amount of SN Dusts

Cas A is one of the most-studied young CC SNRs. It is $\sim 340 \mathrm{yr}$ old and at a distance of $3.4 \mathrm{kpc}$. The basic structure of young SNRs such as Cas A look like Fig. 1. At the outermost boundary, there is the SNR shock propagating into the ambient medium. In the inner region, there is 'reverse shock' propagating back into the $\mathrm{SN}$ ejecta, which is driven by the elevated pressure of the shocked ambient medium. The shocked ambient medium and the shocked SN ejecta are separated by a contact discontinuity where the density and temperature change discontinuously, while the pressure remains the same. The relative distances from the explosion center to the SNR and reverse shocks depend on the properties of the SN ejecta and the ambient medium, as well as the time after the explosion. In Cas A, which is the remnant of SN IIb with a progenitor mass of $\sim 20 M_{\odot}$, the ambient medium is believed to be a slowly-expanding dense red supergiant wind (Chevalier, 2005), and the distances to the SNR and reverse shocks are about $2.6 \mathrm{pc}$ and $1.7 \mathrm{pc}$, respectively (Hwang \& Laming, 2012).

The dust emission could originate from several regions in Fig. 1. In the shocked ambient medium, there could be CS dusts, while in the shocked SN ejecta, there could be SN dusts. They should have survived from the SNR and reverse shocks, respectively. These fresh stardusts in the shocked regions are heated by collisions with electrons in hot plasma, and they are hot $(\sim 100 \mathrm{~K})$ and emit MIR emission. In Cas A, hot SN dusts at $65-265 \mathrm{~K}$ have been detected by Hines et al. (2004) and Rho et al. (2008) using Spitzer. The estimated mass is $0.020-0.054 M_{\odot}$ and it is believed to be SN dusts swept-up by a reverse shock. On the

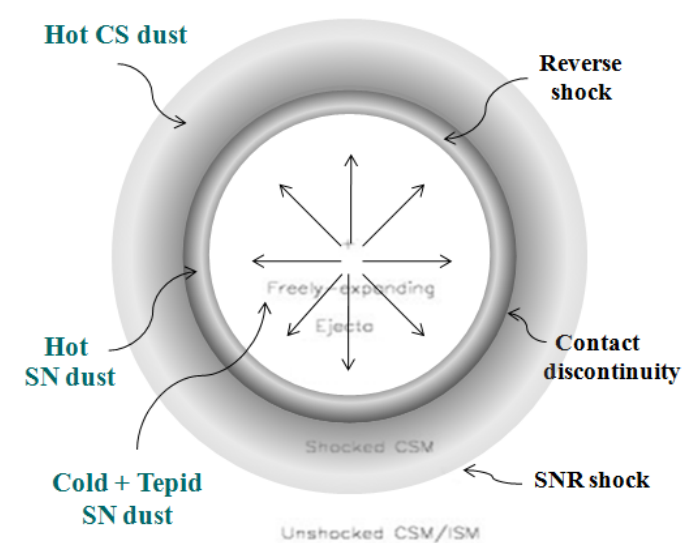

Fig. 1. Structure of young SNRs such as Cas A and the regions where hot and cold/tepid stardusts reside.

other hand, the unshocked SN ejecta dusts in the innermost regions may be heated by X-ray and UV radiation from shocked gas. It is cold and therefore their emission is in the FIR, which is very hard to observe because of contaminating emission from the general interstellar medium (ISM).

In early 2000, it was proposed that there is a large amount of cold SN dusts associated with Cas A, i.e., 2-4 $M_{\odot}$ at $15-20 \mathrm{~K}$ (Dunne et al., 2003). But recent FIR observations by AKARI and Herschel found no supporting evidence for such a large amount (Sibthorpe et al., 2010; Barlow et al., 2010). Instead, Sibthorpe et al. (2010) have carried out a detailed analysis of the AKARI and BLAST data and were able to separate out the FIR emission that might be from the dusts in the SN ejecta. The temperature of this new dust component was $35 \mathrm{~K}$, much lower than that of the shocked dusts but warmer than the cold dusts in the general ISM. The mass of this "tepid" dust component was estimated to be $0.06 M_{\odot}$. Later, Barlow et al. (2010) did high-resolution observations with Herschel infrared space telescope to confirm that the emission is confined to the interior of Cas A as expected. Therefore, the total mass of SN dusts in Cas A is about $0.1 M_{\odot}$, and it is the largest SN dust mass observed in Galactic SNRs.

\subsection{G292.0+1.8: SNR with Prominent CS Dusts}

G292.0+1.8 (MSH 11-54) is a young, $\sim 3,000$ yr-old CC SNR at $6.2 \mathrm{kpc}$. It is one of the so-called Oxygenrich SNRs where the emission from oxygen and neon SN ejecta are prominent. The AKARI MIR and FIR images show two prominent structures; a bright equa- 
torial ring (ER) structure along the east-west direction and an outer elliptical shell structure (Fig. 2; Lee et al., 2009). The ER and the elliptical shell structures were partly visible in the optical and/or X-rays, but they are much more clearly revealed in the AKARI images. There is no evident difference in infrared colors of the two prominent structures, which is consistent with the previous proposition that both structures are of CS origin. The IR morphology of the two structures indicates that the mass injection during the final evolutionary stage of the progenitor was neither symmetric nor continuous. Lee et al. (2009) showed that the spectral energy distribution (SED) of the SNR could be explained by thermal emission from two dust components at $103 \mathrm{~K}$ and $\gtrsim 47 \mathrm{~K}$, respectively. The total dust mass is estimated to be $\lesssim 4.8 \times 10^{-2} M_{\odot}$. Then, by comparing to the total mass $\left(30.5 M_{\odot}\right)$ of X-ray emitting hot gas, they derived dust-to-gas mass ratio of $\lesssim 1.6 \times 10^{-3}$, which was considerably lower than the ratio $6.2 \times 10^{-3}$ in the local ISM. Lee et al. (2009) concluded that either $75 \%$ of the dust in G292.0+1.8 might have been destroyed by the SN shock, or the initial dust-to-gas ratio surrounding G292.0+1.8 might be lower than the local value.

Lee et al. (2009) also detected the MIR emission from the SN ejecta. The SN ejecta are prominent in the AKARI 15/24 $\mu \mathrm{m}$ ratio map because of their high ratios which is at least partly due to the emission lines from Ne ions in the SN ejecta (Fig. 2). Lee et al derived the IR fluxes from the ejecta, and estimated the dust mass of $<4 \times 10^{-4} M_{\odot}$, which is not a significant amount.

\subsection{G54.1+0.3: SNR with CS/IS or SN Dusts?}

G54.1+0.3 is about 3,000 yr old, young SNR at a distance of 6-8 kpc. The remnant had been known as a Plerion or a Crab-like SNR, but recently the SNR shell with a radius of $\sim 10 \mathrm{pc}$ has been detected (Lang et al. 2010). Since there is no indication of strong CSM, it is thought to be a remnant of Type IIP SN (Chevalier, 2005). Using AKARI, Koo et al. (2008) detected an IR loop surrounding the SNR. There are stellar sources with strong IR excesses embedded in the loop. Koo et al. showed that they are OB stars with interstellar (IS) extinction of about $8 \mathrm{mag}$, which is comparable to the extinction to the SNR derived from the X-rays. The spatial coincidence and the comparable extinction support their association with the SNR. The SEDs of the

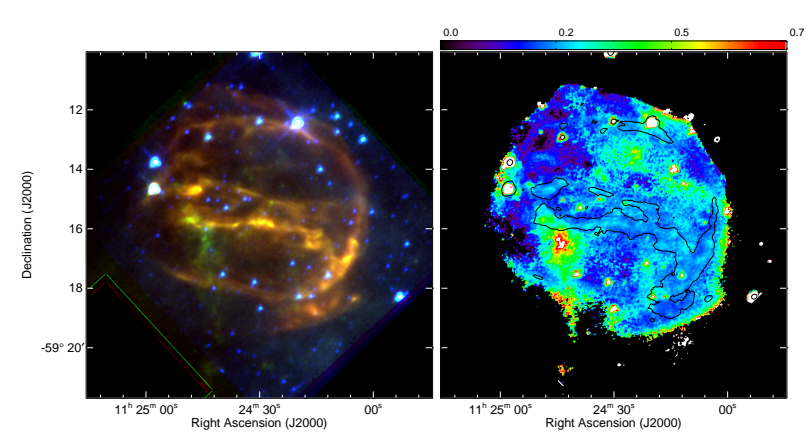

Fig. 2. Left: three-color image of G292.0+1.8 produced from $24 \mu \mathrm{m}(\mathrm{R}), 15 \mu \mathrm{m}(\mathrm{G})$, and $7 \mu \mathrm{m}(\mathrm{B})$. The $15 \mu \mathrm{m}$-bright greenish structure in the southwestern area are the SN ejecta, whereas the prominent, yellowish structure is circumstellar medium (CSM). Right: the background-subtracted $15 / 24 \mu \mathrm{m}$ ratio image. The pixels with low $24 \mu \mathrm{m}$ intensities are blanked out. The bright structures in $24 \mu \mathrm{m}$ images are shown by contour (Lee et al., 2009).

stellar sources after extinction correction have stellar part and MIR/FIR excess, which are similar to those of YSOs without much CSM. And the confinement of these stellar sources in a loop-like structure surrounding the PWN led Koo et al. to propose that they are YSOs whose formation was triggered by the progenitor of the SNR. But later Temim et al. (2010) carried out Spitzer spectroscopy of the IR loop, and detected dust emission with a bump at $21 \mu \mathrm{m}$ which is similar to that in Cas A. This, together with broad emission lines, led them to propose a different scenario where these stars are the members of a cluster that the progenitor belonged, and the IR emission is from SN dusts. So there are two opposed scenarios for the origin of dust. Kim et al. (2012) discussed the pros and cons of the two scenarios based on the near-infrared (NIR) spectra and infrared SEDs of the stellar sources.

Fig. 3 shows the appearance of the IR loop in AKARI, Spitzer, and Herschel images, and Fig. 4 shows the SED of the IR shell. The AKARI FIS, Herschel PACS 70 and $160 \mu \mathrm{m}$, and SPIRE 250 and 350 $\mu \mathrm{m}$ fluxes are derived in this work. These long wavelength data points are important because they constrain the mass of cold dusts. If we fit the SED with two-temperature components assuming silicate dusts, we obtain dust masses of $1.3(0.6) \times 10^{-2} M_{\odot}$ at $73 \mathrm{~K}$ and $0.87(0.08) M_{\odot}$ at $32 \mathrm{~K}$ for hot and cold dusts, re- 


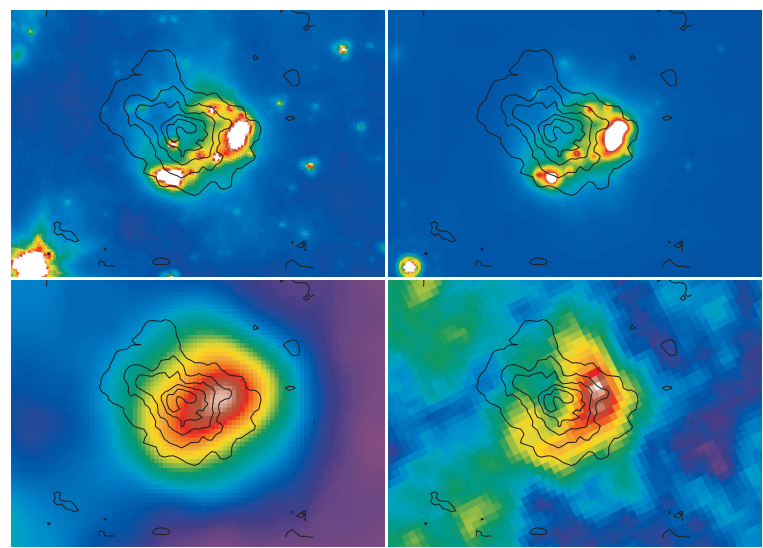

Fig. 3. Clockwise from top left: AKARI $15 \mu \mathrm{m}$, Spitzer $24 \mu \mathrm{m}$, Herschel PACS $70 \mu \mathrm{m}$, and AKARI $90 \mu \mathrm{m}$ images of the IR loop in G54.1+0.3. VLA $4.87 \mathrm{GHz}$ radio contours are overlaid. Contour levels increase toward the center of the SNR.

spectively. The distance is assumed to be $7 \mathrm{kpc}$. For graphite dusts, they are $6.4(1.8) \times 10^{-3} M_{\odot}$ at $89 \mathrm{~K}$ and $0.58(0.06) M_{\odot}$ at $31 \mathrm{~K}$. If the emission is from SN dusts, this will be the largest SN dust mass detected in Galactic SNRs so far. On the other hand, it may indicate that this cold component is of IS or CS origin. This is a preliminary result and a detailed modeling considering the distribution of IR emission is needed.

\subsection{MSH 15-52: SNR with Stardusts near Pulsar}

The SNR MSH 15-52 is a young SNR of complex morphology. In radio and X-ray, the entire structure occupies almost $40 \mathrm{pc}$ at a distance of $4 \mathrm{kpc}$. On the other hand, there is a young pulsar B1509-58 with a characteristic age of 1,700 yrs that is at the center. Gaensler (1999) made a detailed study of this remnant and based on the large extent, in spite of its young age, he proposed that this might be the remnant of a Type Ib SN where the progenitor created a large bubble during the Wolf-Rayet (WR) phase before exploding. He further speculated that it could have been a binary system with the O star called Muzzio 10, which is only $15^{\prime \prime}$ apart. There is a bright, mid-infrared compact source (IRAS 15099-5856) located close to the pulsar B1509-58. Arendt (1991) investigated the properties of this source and concluded that it is heated either by hot plasma associated with the SNR or by the O star Muzzio 10.

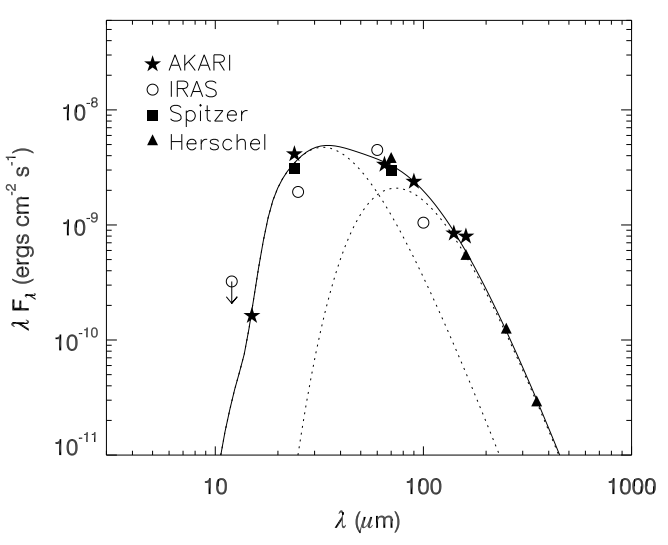

Fig. 4. SED of the IR loop in G54.1+0.3 and a model fit assuming silicate dusts at two different temperatures.

AKARI observed this SNR in six AKARI IRC bands and the images were quite spectacular; it is composed of a bright central compact source (hereafter IRS1) surrounded by a complex network of filaments (Fig. 5; Koo et al., 2011). The unique feature of IRAS 15099-5856 is its prominent crystalline silicate emission. The Spitzer IRS MIR spectrum of IRS1 shows prominent emission features of Mg-rich crystalline silicates, i.e., $\mathrm{Mg}_{2 x} \mathrm{Fe}_{2-2 x} \mathrm{SiO}_{4}$ with $x \sim 1$, which indicates that the dust in IRS1 is of CS origin. The spectrum at wavelengths longer than $15 \mu \mathrm{m}$ is indeed very similar to the Herbig Be star HD 100546 and the luminous blue variable (LBV) R71 (see Fig. 4 in Koo et al., 2011). However, the core of IRS1 is extended $\left(9 .^{\prime \prime} 6 \times 5 .^{\prime \prime} 1\right.$ or $\left.0.19 \times 0.10 \mathrm{pc}\right)$ and there are no stellar sources $\left(<20^{\text {th }} \mathrm{mag}\right.$ at $\left.2.15 \mu \mathrm{m}\right)$ associated with it, which led Koo et al. to conclude that IRS1 is heated by UV radiation from the nearby ( $13^{\prime \prime}$ or $\left.0.25 \mathrm{pc}\right) \mathrm{O} 4.5$ III star Muzzio 10, as was speculated by Arendt (1991), and that its crystalline silicates most likely originate in a mass outflow from the progenitor of the SNR MSH1552. They further proposed that IRS1 could have survived the SN blast wave because the progenitor and Muzzio 10 formed a close binary system, as first proposed by Gaensler et al. (1999), and because Muzzio 10 shielded IRS1 from the SN blast wave. The close proximity of Muzzio 10 and the pulsar B1509-58 supports this possibility. The enhanced abundance of crystalline silicates is also consistent with this scenario since in binary stars, grain growth and crystalline formation can be enhanced through circumbinary disks (Gielen 


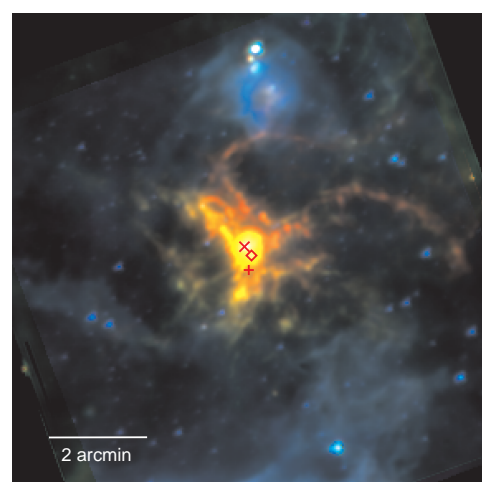

Fig. 5. Three-color image of IRAS 15099-5856 produced from AKARI S11 (blue), L15 (green), and L24 (red) images. The cross marks the peak position of IRS1, while the plus and diamond signs mark the positions of the pulsar B1509-58 and the O star Muzzio 10, respectively (Koo et al., 2011).

et al., 2008). IRS1 is most likely the relic of a massive star death in a close binary system, as shaped by the supernova, the pulsar wind, and the intense ionizing radiation of the embedded $\mathrm{O}$ star.

Fig. 6 is a schematic diagram showing the configuration before and after the explosion. Before the explosion, the dusts might have been confined into a disk-like or a shell-like structure, the detailed structure of which depends on the mass-loss history of the progenitor. For massive binaries, for example, the dusts formed by colliding winds could be confined to a spiral ring structure as in the binary Wolf-Rayet star system WR 104 (Tuthill et al., 2008). When the SN exploded, all materials in the disk might have been swept-up by the SN blast wave except the ones in the area shielded by the companion $\mathrm{O}$ star. If a significant fraction of the mass loss that led to the formation of IRS1 were distributed in a disk, then the total dust mass of the disk would be about $(2 \pi \times 0.6 \mathrm{pc}) /(0.2 \mathrm{pc})$ times the mass of IRS1 or about $0.17 M_{\odot}$, where $0.6 \mathrm{pc}$ is the distance to IRS1 from the explosion center and $0.2 \mathrm{pc}$ is the size of IRS1. For a gas-to-dust ratio of 100 , this implies a total mass of $17 M_{\odot}$, which is large but not unreasonable. Further observations are needed to confirm whether IRS1 is indeed a fragment of dusty shell/disk around a massive progenitor that had survived from the SN blast wave.
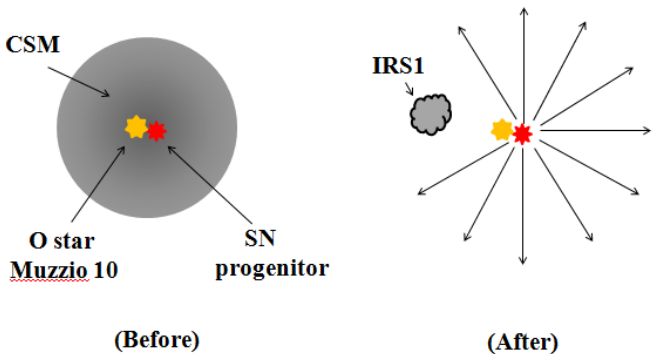

Fig. 6. (a) Schematic diagram showing the configuration before the SN explosion. The CSM may be confined to a disk-like structure around the binary progenitor. (b) After the explosion, all CSM have been swept up by the SN blast wave except the material, i.e., IRS1, that had been shielded by the companion star Muzzio 10.

\subsection{CS Dusts in SNRs in Transition Phase}

A result on the dusts in SNRs in an earlier stage has been recently reported by Tanaka et al. (2012). They have searched IR emission associated with six extragalactic SNRs. These remnants are 10- to 100- years old, so that we may say that they are in transition phase from SNe to SNRs. They detected dust emission in one of them, 1978k. SN 1978k is an SN IIn, which means that its spectra show narrow absorption lines indicating large amount of CS material. Its progenitor is thought to be LBVs. They investigated possible origins for the IR emission, and concluded that it is most likely from the shocked CS dusts, not from SN dusts or IS dusts heated by the SN flash. The derived dust mass was $1.3 \times 10^{-3} M_{\odot}$ at $230 \mathrm{~K}$. They showed that these SNRs in transitional phase will provide a good opportunity for future instruments to study the CS/IS environment of SN and also SN dusts at longer wavelengths.

\section{CONCLUDING REMARK}

Recent observations by the space infrared telescopes AKARI, Spitzer, and Herschel revealed stardusts associated with young CC SNRs. Their infrared characteristics and physical/chemical properties are diverse. The AKARI results presented in this paper show part of the diversity. Presumably such diversity originates from different SN types, i.e., different physical/chemical stellar structures and CS environments at the time of explosion. The diverse types of extragalactic SNe indi- 
cate that there is a variety of channels to the explosion, probably depending on metallicity, rotation rate, mass loss rate, and binarity or multiplicity. The CS environment might also vary considerably in different SN types, as implied by the observed diverse mass-loss patterns in evolved massive stars. The questions that we want to address might be; What dust species and how much dusts form in each SN type? How effective each SN type is in processing CS and IS dusts? What's the population of different SN types, and what's their contribution to the dust inventory and what's their role in the dust life cycle in galaxies? The SNRs in the Galaxy and nearby galaxies have their own advantages in addressing these questions, and we look forward to seeing more exciting results in future.

\section{ACKNOWLEDGEMENTS}

I wish to thank all people involved in the AKARI SNR project. I thank Kim, H.-J. who helped in the estimation of the FIR fluxes of the IR loop in G54.1+0.3. This research was supported by Basic Science Research Program through the National Research Foundation of Korea (NRF) funded by the Ministry of Education, Science and Technology (NRF-2011-0007223).

\section{REFERENCES}

Arendt, R. G., 1991, IRAS 15099-5856 and the Composite Supernova Remnant G320.4 - 1.2, ApJ, 101,2160

Barlow, M. J., et al., 2010, A Herschel PACS and SPIRE Study of the Dust Content of the Cassiopeia a Supernova Remnant, A\&A, 518, L138

Chevalier, R. A., 2005, Young Core-Collapse Supernova Remnants and Their Supernovae, ApJ, 619, 839

Dunne, L., Eales, S., Ivison, R., Morgan, H., \& Edmunds, M., 2003, Type II Supernovae as a Significant Source of Interstellar Dust, Nature, 424, 285

Draine, B. T., 2009, Interstellar Dust Models and Evolutionary Implications, in Cosmic Dust - Near and Far, 414, 453

Gaensler, B. M., Brazier, K. T. S., Manchester, R. N., Johnston, S., \& Green, A. J., 1999, SNR G320.401.2 and PSR B1509-58: New Radio Observations of a Complex Interacting System, MNRAS, 305,724

Gielen, C., van Winckel, H., Min, M., Waters, L. B. F. M., \& Lloyd Evans, T., 2008, SPITZER Survey of Dust Grain Processing in Stable Discs around Binary Post-AGB Stars, A\&A, 490, 725

Hines, D. C., et al., 2004, Imaging of the Supernova Remnant Cassiopeia A with the Multiband Imaging Photometer for Spitzer (MIPS), ApJS, 154, 290

Hwang, U. \& Laming, J. M., 2012, A Chandra X-Ray Survey of Ejecta in the Cassiopeia A Supernova Remnant, ApJ, 746, 130

Kim, H.-J., et al., 2012, in preparation.

Koo, B.-C., et al., 2008, A Massive-Star-forming Infrared Loop around the Crab-like Supernova Remnant G54.1+0.3: Post-Main-Sequence Triggered Star Formation?, ApJ, 673, L147

Koo, B.-C., 2009, AKARI Observations of Supernova Remnants, in AKARI, a Light to Illuminate the Misty Universe, ASPC, 418, 167

Koo, B.-C., et al., 2011, IRAS 15099-5856: Remarkable Mid-infrared Source with Prominent Crystalline Silicate Emission Embedded in the Supernova Remnant MSH15-52, ApJ, 732, 6

Lang, C. C., Wang, Q. D., Lu, F., \& Clubb, K. I., 2010, The Radio Properties and Magnetic Field Configuration in the Crab-Like Pulsar Wind Nebula G54.1+0.3, ApJ, 709, 1125

Lee, H.-G., et al., 2009, AKARI Infrared Observations of the Supernova Remnant G292.0+1.8: Unveiling Circumstellar Medium and Supernova Ejecta, ApJ, 706, 441

Matsuura, M., et al., 2011, Herschel Detects a Massive Dust Reservoir in Supernova 1987A, Science, 333, 1258

Nozawa, T., et al., 2010, Formation and Evolution of Dust in Type IIb Supernovae with Application to the Cassiopeia A Supernova Remnant, ApJ, 713, 356

Rho, J., et al., 2008, Freshly Formed Dust in the Cassiopeia A Supernova Remnant as Revealed by the Spitzer Space Telescope, ApJ, 673, 271

Shinn, J.-H., Koo, B.-C., Seon, K.-I., \& Lee, H.-G., 2011, AKARI Near-infrared Spectral Observations of Shocked H2 Gas of the Supernova Remnant IC 443, ApJ, 732, 124

Sibthorpe, B., et al., 2010, AKARI and BLAST Observations of the Cassiopeia A Supernova Remnant and Surrounding Interstellar Medium, ApJ, 719,1553

Tanaka, M., et al., 2012, A Search for Infrared Emission from Core-collapse Supernovae at the Transitional Phase, ApJ, 749, 173

Tuthill, P. G., et al., 2008, The Prototype CollidingWind Pinwheel WR 104, ApJ, 675, 698 